\title{
Estrategias de Producción Más Limpia para el Adecuado Manejo y Reducción en el Origen de Residuos Peligrosos: Caso de Estudio Industrias Litográficas y Tintorerías
}

\author{
Cleaner production strategies to correct management and source reduce of \\ hazardous waste: study case drycleaner and lithograph industries
}

\author{
Janneth Cubillos Vargas¹, Yenire A. González Moreno ${ }^{1}$, Adriana M. Ruiz Sánchez ${ }^{1}$, María Eugenia \\ Vélez Riaño ${ }^{2}$, Diego Paredes Cuervo ${ }^{1}$ \\ ${ }^{I}$ Grupo de Investigación en Agua y Saneamiento, Facultad de Ciencias Ambientales, Universidad \\ Tecnológica de Pereira, Colombia \\ ${ }^{2}$ Grupo de Investigación en Gestión Ambiental de la Corporación Autónoma Regional de Risaralda \\ Correo: jacubillos@utp.edu.co, yagonzalez@utp.edu.co, amruiz@utp.edu.co, \\ mvelez@carder.gov.co, diparede@utp.edu.co
}

\begin{abstract}
Resumen - En el sector industrial cada día, existe una creciente necesidad de reconocer el tipo y cantidad de residuos sólidos y particularmente los residuos sólidos peligrosos-RESPEL que se generan, así como los impactos negativos que el mal manejo de éstos puede originar, con el fin de producir herramientas para la formulación de estrategias de manejo apropiado de la salud pública, protección ambiental y las normas actuales del país.
\end{abstract}

El presente artículo sustenta la evaluación de los subsectores de litografías y tintorerías, que representan un impacto en la generación y manejo de residuos con características potencialmente peligrosas en los municipios de Pereira y Dosquebradas.

Palabras clave - Residuos Peligrosos, Tintorerías, Litografías, Producción más limpia

Abstract - In the industrial sector every day, there is a growing need to recognize the type and quantity of solid waste that is generated as well as the negative impacts that their mismanagement can produce, in order to have tools for the strategies formulation of proper handling and to comply with environmental protection, public health and the current regulatory framework of our country.

This article supports the evaluation of lithographs and Drycleaners subsectors that represent an impact on the generation and management of hazardous waste in the municipalities of Pereira and Dosquebradas.

Key Word -Hazardous waste, Drycleaner, lithograph, Cleaner production

\section{INTRODUCCIÓN}

La generación de residuos sólidos a escala mundial se ha convertido en una gran problemática ambiental, dado el inadecuado manejo que se ofrece en cuanto al aprovechamiento, tratamiento y disposición final de los mismos. Esta situación se agudiza al existir altos patrones de consumo y una mayor producción e incorporación de compuestos químicos, sintéticos y elementos electrónicos que dirigen el avance tecnológico de la sociedad, dando origen a residuos de difícil degradación, que a la postre son incorporados en el ambiente sin un adecuado manejo.

De este modo, surge el concepto de "residuos peligrosos", que según el decreto 4741 de 2005 [1] son aquellos que "por sus características corrosivas, reactivas, explosivas, tóxicas, inflamables, infecciosas o radiactivas pueden causar riesgo o daño para la salud humana y el ambiente. Así mismo, los envases, empaques y embalajes que hayan estado en contacto con ellos". Actualmente, el control de éste tipo de residuos en Colombia, es aún incipiente por la falta de conocimiento sobre sus aspectos técnicos y legales por parte de los generadores, comercializadores, receptores, autoridades, academia y comunidad en general.

En los países en desarrollo la atención a la problemática asociada a los residuos peligrosos o RESPEL, ha sido un poco más lenta al ser comparada con los países desarrollados, encontrándose falencias en la disponibilidad de infraestructura adecuada para cada contexto, que permitan realizar una gestión apropiada para dichos residuos. Esta situación ha potencializado el manejo inadecuado de los desechos y el

Fecha de Recepción: 04 de Junio de 2014

Fecha de Aceptación: 11 de Noviembre de 2015 
incremento de impactos ambientales por disposición inadecuada [2].

Actualmente en Colombia, se cuenta con el aplicativo Registro de Generadores RESPEL, como una herramienta para cuantificar la generación y el manejo anual o gestión de los residuos sólidos peligrosos, en el cual el generador solicita la inscripción ante la autoridad ambiental competente, si cumple una de las clasificaciones por tamaño: gran generador cuando la cantidad de RESPEL producida es mayor a 1000 $\mathrm{kg} / \mathrm{mes}$; mediano, si la cantidad de RESPEL está entre 100 $1000 \mathrm{~kg} / \mathrm{mes}$ y pequeño generador, si está entre $10-100$ $\mathrm{kg} / \mathrm{mes}[1]$.

La información sobre los RESPEL en el país es registrada en el Sistema de Información Ambiental del IDEAM, por las autoridades ambientales, quienes administran el (los) aplicativo (s) en su jurisdicción. Del sistema se obtienen las estadísticas relacionadas con la producción, tipo de residuos peligrosos generados y actividades productivas generadoras para el país. Por ejemplo, en el año 2010 se reportó para Colombia una producción de 138.090 toneladas de RESPEL [3], los cuales en su mayor proporción han sido orientados más hacia el tratamiento y disposición final que hacia la prevención y minimización de la generación.

Según Martínez y Rozo [4], aunque el registro de generadores permite tener una idea sobre la producción, manejo y disposición final de residuos peligrosos, tanto el IDEAM como las Corporaciones Ambientales infieren que ésta no es la realidad del país, principalmente porque aún existe un gran desconocimiento en la temática, no se sabe a ciencia cierta dónde se generan estos residuos al interior de los procesos productivos y cómo están siendo almacenados, tratados y/o dispuestos, ya que la información registrada no es consecuente con lo evidenciado en campo.

En Risaralda, se han identificado en el Plan Departamental de RESPEL [5] cerca de 484 generadores en los 14 municipios, obedeciendo a una producción de $133.453 \mathrm{~kg} / \mathrm{mes}$, distribuidos en los sectores productivos: minero energético, servicios, comercial, industrial, oficial, agropecuario, educativo e institucional; no obstante, no se tiene control real respecto al tipo de residuos generados, cantidad y manejo que se da a los mismos.

Para abordar ésta problemática, se proponen opciones de mejora en la gestión de los residuos peligrosos, estimando que entre las mejores está la reducción de su producción a través de prácticas de producción más limpia, y en lo posible, adelantar acciones reciclaje, recuperación o aprovechamiento [6].

Con la finalidad de prevenir y minimizar la generación de desechos peligrosos, la Autoridad Ambiental de Risaralda a través del Plan Departamental de Residuos Peligrosos (RESPEL) [5] consideró al igual que la Política Nacional de RESPEL [6], una línea de acción para la promoción e implementación de estrategias de producción más limpia, dirigida a encontrar soluciones técnicas que promuevan el desarrollo sostenible de la región y la protección del medio ambiente.

La producción más limpia es vista como herramienta preventiva que toma importancia en los diferentes sectores industriales, ya que evita la implementación de soluciones a final de tubo, reduciendo la generación del contaminante en la fuente, optimizando el consumo de materias primas y de recursos naturales; $\mathrm{y}$, disminuyendo costos de tratamiento y disposición final de los desechos generados [7]. Según el Programa de las Naciones Unidas para el Medio AmbienteUNEP [8], la aplicación de mecanismos de producción más limpia permite llegar a la ecoeficiencia y reducir riesgos para el ser humano y el ambiente.

Con base en esto, el objetivo principal del presente estudio es proponer estrategias de producción más limpia (PML) y de manejo adecuado de los RESPEL para las actividades de acabados de productos textiles y de impresión, de los subsectores tintorerías y litografías, bajo el enfoque de buenas prácticas de producción y cambios o mejoras tecnológicos.

\section{MARCO GEOGRÁFICO DEL ÁREA DE ESTUDIO}

El estudio se realizó en el Departamento de Risaralda en los municipios de Pereira y Dosquebradas, ubicados en la Subregión I (Vertiente Oriental del Río Cauca). En esta Subregión se concentra el mayor desarrollo urbano e industrial del Departamento y por ello se resalta el interés para la Corporación Autónoma Regional de Risaralda en ejercer inspección, vigilancia y control a las actividades comerciales e industriales de los municipios, en cuanto a la generación de residuos peligrosos.

\section{METODOLOGÍA}

El estudio se desarrolló a través de tres fases específicas:

\section{A. Priorización y selección de sectores productivos}

La priorización y selección de los sectores productivos generadores de RESPEL a nivel regional, se llevó a cabo a través de la revisión detallada del Plan Departamental de RESPEL, el Registro de Generadores de RESPEL de la CARDER y de reuniones conjuntas entre el personal encargado del área de residuos sólidos de la Corporación Autónoma Regional de Risaralda y del Grupo de Investigación en Agua y Saneamiento de la Universidad Tecnológica de Pereira (ejecutor del proyecto).

A partir de estas reuniones, se estableció que debido a las características de peligrosidad de los insumos químicos utilizados para el desarrollo de sus procesos y el poco conocimiento existente respecto a la gestión de tales desechos peligrosos en la región, los sectores de litografías y tintorerías serían aquellos de mayor interés, ya que si bien son empresas pequeñas, se encuentran en gran cantidad en los municipios de 
Pereira y Dosquebradas, dando origen a volúmenes representativos de RESPEL cuya gestión debe ser monitoreada.

\section{Evaluación del sector productivo}

Una vez elegidos los sectores productivos a trabajar, se identificó el número de empresas por cada sector con la ayuda del registro de generadores de RESPEL; a través de un taller de socialización, se evidenció el interés de participación, vinculándose 11 litografías y 8 tintorerías para la evaluación de la gestión de los residuos peligrosos generados en sus procesos productivos.

El trabajo de campo se efectuó con aplicación de encuestas estructuradas y entrevistas personalizadas para recolectar información detallada referente a: etapas productivas (descripción del proceso, flujogramas), insumos utilizados y residuos generados (características de peligrosidad). También se identificó la gestión interna y externa efectuada actualmente por el sector (rotulado, almacenamiento, transporte, tratamiento, disposición final, entre otros), existencia e implementación del plan de gestión de residuos peligrosos y su inscripción como generador de RESPEL ante la autoridad ambiental, en caso de aplicar.

La caracterización de peligrosidad de los residuos generados en los subsectores, se realizó con la revisión de las etiquetas de los productos químicos que en algunos casos contiene impreso el rombo NFPA (National Fire Protection Association), que brinda información relativa de los riesgos por toxicidad, inflamabilidad, reactividad y riesgos específicos de la sustancia. Adicionalmente, se revisaron las fichas u hojas de seguridad de los insumos, para obtener información específica referente a: tipificación de peligros, medidas contra incendios, propiedades fisicoquímicas, información ecológica y consideraciones de eliminación de los residuos.

Igualmente, se revisaron los anexos I, II y III del decreto 4741 de 2005 que establece la peligrosidad de los residuos por procesos, corrientes o características peligrosas. Los desechos que no se encontraban listados dentro de los anexos I y II, se tipificaron en compañía del personal de la Corporación Autónoma Regional de Risaralda (CARDER), de acuerdo a las hojas de seguridad de los insumos utilizados, estableciendo si el desecho era considerado tóxico, inflamable, corrosivo o reactivo.

2. Formulación de estrategias de producción más limpia

Las estrategias de producción más limpia se formularon con base en el análisis de los procesos productivos de las litografías y las tintorerías, en las que se incluyeron alternativas de buenas prácticas, cambios y/o mejoras tecnológicas tal como se ilustra en la figura 1.

Las alternativas de cambios en procesos y sustitución de materias primas e insumos no fueron tenidas en cuenta, debido a que los procesos realizados en las tintorerías y litografías son estandarizados. Adicionalmente, para sugerir modificaciones en los insumos se debe conocer muy bien el proceso productivo, ya que el cambio de un tipo o cantidad de material puede afectar la calidad esperada del producto; por esta razón las empresas mantienen un estricto control respecto a las materias primas que utilizan y solicitan a sus proveedores información sobre las ventajas y desventajas de la aplicación de nuevos materiales, conociendo de esta forma cuáles son los insumos convenientes para su proceso.

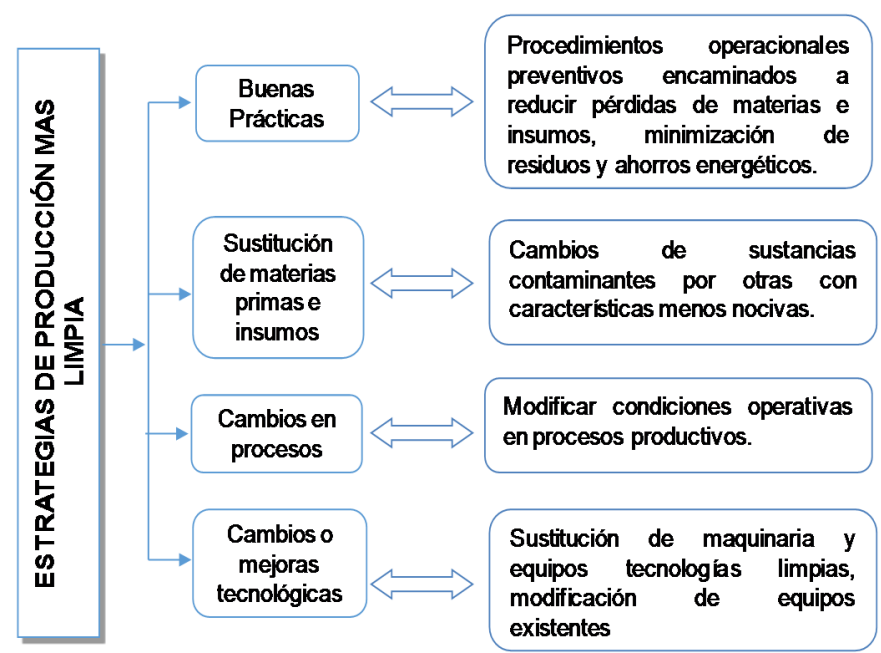

Fig. 1. Diagrama de Estrategias de Producción Más Limpia (PML). Fuente: [7, 9].

A continuación se definen las estrategias de PML que se tendrán en cuenta en el desarrollo del trabajo:

- Buenas prácticas: son medidas relacionadas con prevenir la pérdida de materias primas, la minimización de residuos, el ahorro de agua, energía y el mejoramiento de la empresa. Son acciones voluntarias que se puedan aplicar con el objetivo de Racionalizar, Reducir, Reutilizar y/o Reciclar y mejorar las condiciones de trabajo, de la salud y seguridad ocupacional en la empresa [7].

- Cambios o mejoras tecnológicas: consiste en la sustitución de maquinaria y equipos de tecnología que presenta baja eficiencia ambiental, baja productividad o baja eficiencia en la cantidad y calidad del producto, por nuevos equipos y maquinaria con tecnología de punta que pueda reducir el consumo de insumos, materia prima y energía; minimizar la generación de cargas contaminantes y obtener productos de alta calidad [7].

\section{RESULTADOS}

En la evaluación de los subsectores de interés, se encontró las siguientes características asociadas a la gestión de RESPEL:

A. Litografías subsector de la industria gráfica. 
Las litografías tienen una alta participación como pequeñas y medianas empresas con actividades y procesos, que van desde la preparación de materiales hasta la elaboración de productos debidamente terminados como periódicos, revistas, libros, materiales de publicidad y empaques. [10].

La importancia ambiental que se adjudica al sector se representa en la necesidad de controlar las emisiones atmosféricas, asociadas al manejo de solventes, el manejo de los residuos líquidos industriales provenientes de los procesos de revelado y la gestión integral de los residuos sólidos que involucra desde la presencia de elementos reciclables hasta la generación de residuos peligrosos [11].

La tabla 1 presenta los residuos generados durante la evaluación de la producción gráfica, al interior de cada etapa de su proceso.

Tabla 1. Producción de RESPEL por etapa del proceso litográfico

\begin{tabular}{|c|l|}
\hline Etapa & \multicolumn{1}{|c|}{ Residuos generados } \\
\hline \multirow{4}{*}{ Diseño } & Residuos de Aparatos y Equipos Electrónicos \\
\cline { 2 - 3 } & Toner de impresoras \\
\cline { 2 - 2 } & Papel \\
\hline \multirow{4}{*}{ Preprensa } & Líquido de revelado \\
\cline { 2 - 2 } & Líquido fijador \\
\cline { 2 - 2 } Prensa o & Películas usadas \\
\hline \multirow{4}{*}{ impresión } & Planchas de impresión \\
\cline { 2 - 2 } & Desechos de papel \\
\cline { 2 - 2 } & $\begin{array}{l}\text { Paños y recipientes plásticos contaminados con } \\
\text { sustancias químicas }\end{array}$ \\
\cline { 2 - 2 } & Lodos de tinta \\
\hline \multirow{4}{*}{ Acabado } & Recipientes de barniz y pegamento blanco \\
\cline { 2 - 2 } & Paños contaminados \\
\cline { 2 - 2 } & Residuos de Papel foil \\
\hline
\end{tabular}

Una vez identificados los diversos residuos producidos, se siguió la metodología planteada, analizando los insumos utilizados en cada etapa del proceso litográfico, usando el rombo NFPA, las hojas de seguridad de los mismos y las listas de los residuos presentados en la tabla 1 y los anexos I, II y III del Decreto 4741 de 2005, estableciendo que excepto el papel, la totalidad de los residuos sólidos allí originados son de características peligrosas por su potencial inflamable, corrosivo y tóxico y por lo tanto, exigen una gestión especializada: interna (en la empresa) y externa a ellas (gestores externos).

Los principales tipos de RESPEL identificados fueron: Y6 (Desechos resultantes de la producción, la preparación y la utilización de disolventes orgánicos), Y12 (Desechos resultantes de la producción, preparación y utilización de tintas, colorantes, pigmentos, pinturas, lacas o barnices), Y13 (Desechos resultantes de la producción, preparación y utilización de resinas, látex, plastificantes o colas y adhesivos) y Y16 (Desechos resultantes de la producción; preparación y utilización de productos químicos y materiales para fines fotográficos).
1. Manejo interno de RESPEL en el subsector litográfico

De acuerdo con los resultados de información recopilada en las litografías visitadas, el $100 \%$ de las empresas realizan algunas labores de separación en la fuente de los residuos generados, los cuales son almacenados en recipientes químicamente inatacables por la sustancia que contienen. Sin embargo, no todas las áreas de almacenamiento cumplen con los requisitos de la normatividad colombiana:

- Disponibilidad de extintores.

- Señalización adecuada.

- Cerramiento de las áreas.

- Implementación y uso de la matriz de compatibilidad de residuos peligrosos.

- Sistema de contención para derrames.

En el trabajo litográfico se originan lodos de tinta que requieren un manejo especial; solo el $9 \%$ de las empresas ha implementado sistemas de lavado en seco para su recolección; no se maneja adecuadamente la rotulación de los recipientes donde se disponen los lodos y por lo tanto, no se resaltan las características de peligrosidad de estos desechos; solo el $64 \%$ de las empresas que generan residuos de lodos, reutilizan los recipientes plásticos impregnados de sustancias químicas para re-envasar este residuo. No obstante, todos los lodos generados son entregados a un gestor externo para la disposición final del residuo, quien tiene una frecuencia de recolección que varía entre quincenal y mensual.

Por otro lado, el 55\% de las litografías entregan los recipientes plásticos que contienen los insumos químicos a un gestor externo autorizado para la recolección, transporte y disposición final y el $45 \%$ restante, vende los recipientes como reciclaje o los disponen como residuos ordinarios, contaminando los otros residuos e imposibilitando la recuperación o reuso.

En las labores de limpieza y mantenimiento de las máquinas, una práctica muy común es el uso de esponjas y paños, los cuales quedan impregnados de grasa, tintas y sustancias químicas que los convierten en RESPEL. El 18\% de las litografías entregan estos desechos al carro recolector de residuos ordinarios del municipio, sin reconocer la necesidad de darles un manejo especial. Igualmente, las empresas que utilizan presurizados en su proceso productivo (64\%), envía el $71 \%$ de estos, junto con los residuos comunes llegando al relleno sanitario.

De las empresas visitadas, el $82 \%$ tiene Plan de Gestión Integral de Residuos Peligrosos (PGIRS-RESPEL), pero en su mayoría, se falla en la implementación por desconocimiento del contenido del plan y por tanto prevalece la realización de prácticas inadecuadas de separación en la fuente, mal almacenamiento y disposición final de los residuos.

El diligenciamiento de la informacion sobre RESPEL lo hacen éstas empresas a través del Registro Único Ambiental (RUA) del IDEAM, el cual también adminsitra la Corporacion 
Ambiental. En la mayoría de los casos las litografías no cuentan con un dato exacto de la cantidad de RESPEL generados al mes, ni clasificación por tipo de residuos, sólo se maneja el reporte - en kilogramos, medido por el gestor externo. El rango en peso de los RESPEL que produce el sector, depende del tamaño de la litografía, variando en los evaluados entre 3 y $180 \mathrm{Kg} / \mathrm{mes}$. Sólo dos establecimientos, reportaron cantidades de residuos peligrosos mensuales mayores a $100 \mathrm{~kg}$. La ausencia de cuantificación por parte de las empresas acerca de los desechos que entregan a los gestores externos, no permite establecer trazabilidad de la gestión de dichos residuos como lo define la norma.

Contrario a las deficiencias en el manejo de los residuos, las litografías han tratado de implementar acciones que permitan reducir impactos al medio ambiente y optimizar su proceso, mediante algunos cambios en insumos y tecnología. En total 7 litografías (64\% de las visitadas), han realizado cambios en la materia prima utilizada para la impresión, en especial en las tintas y en los productos para el lavado de las máquinas litográficas. Los cambios en las tintas se han efectuado porque el mercado ofrece nuevos insumos con propiedades de secado más rápido. Los ensayos con el uso de tintas ecológicas no representó un beneficio para la actividad, ya que se reducía la calidad en la impresión, incumpliendo estandares de la norma ISO 9001.

Igualmente, se ha pasado de tener máquinas impresoras monocolor y bicolor, a máquinas de cuatro colores que permiten hacer mayor número de impresiones en períodos de tiempo más corto y con menos desperdicio de insumos. También se han realizado cambios en el sistema de humectación de las máquinas impresoras con el fin de reducir la generación de los lodos de tinta.

El 73\% de las empresas visitadas realiza buenas prácticas de producción resultado de las capacitaciones impartidas al personal sobre uso, buen manejo de las tintas y de los paños limpiadores. También se programan impresiones continuas para evitar el lavado de las máquinas litográficas y el desperdicio de insumos. Adicionalmente, los residuos de papel que salen del proceso en algunas ocasiones se vende como reciclaje o se reuliza para elaborar las bases de las bolsas de papel.

Algunas de las litografias (27\%) reconocen que identifican insumos que podían ser remplazados en el proceso productivo como son las películas plásticas para la impresión flexográfica (PET), tintas a base de agua y tintas ecológicas; sin embargo, en su mayoría desconocen los beneficios que podrían traer estos productos al ser aplicados por el subsector. Además, se resalta que tienen costos elevados en comparación con los utilizados actualmente, lo que no estimula su implementación.

\section{B. Tintorerías subsector de la industria textil}

La industria manufactura textil es el nombre que se le da al sector de la economía dedicado a la producción de telas, hilos, fibras y ropa. Dentro de este sector se desglosan diferentes subsectores entre los que se encuentran las tintorerías, que involucra procesos de tinción y de mejora de las características de la hilaza, telas, cintas rígidas y elásticas mediante procedimientos físicos y químicos [12].

Las tintorerías son el subsector donde se genera la mayor cantidad de residuos, y especialmente de residuos peligrosos, debido al manejo de insumos que involucran colorantes, químicos y reactivos para los procesos de pre-tratamiento limpieza, descrude, blanqueo, teñido y acabado de las prendas [13]. Las etapas que se desarrollan en el proceso y los respectivos residuos generados en cada una, se observan en la tabla 2.

Tabla 2. Producción de RESPEL por etapa del proceso textil

\begin{tabular}{|c|c|}
\hline Etapa & Residuos generados \\
\hline \multirow{3}{*}{ Prelavado } & Agua residual \\
\hline & Lanas del canal de desagüe \\
\hline & Bolsas contaminadas con sustancias químicas \\
\hline \multirow{5}{*}{$\begin{array}{c}\text { Stone } \\
\text { (Envejecimiento } \\
\text { textil) }\end{array}$} & Agua residual \\
\hline & Bolsas contaminadas con sustancias químicas \\
\hline & Estopas \\
\hline & Residuos de piedra pómez \\
\hline & Lanas del canal de desagüe. \\
\hline \multirow{4}{*}{$\begin{array}{c}\text { Bleach } \\
\text { (Blanqueo) }\end{array}$} & Agua residual \\
\hline & Bolsas contaminadas con sustancias químicas \\
\hline & Estopas del Mangatex \\
\hline & Lanas del canal de desagüe \\
\hline \multirow{3}{*}{$\begin{array}{c}\text { Frosted (pérdida } \\
\text { mecánica de } \\
\text { color) }\end{array}$} & Estopas de la piedra pómez \\
\hline & Bolsas contaminadas con sustancias químicas \\
\hline & Residuos de piedra pómez \\
\hline \multirow{4}{*}{ Teñido } & Agua Residual \\
\hline & Bolsas contaminadas con sustancias químicas \\
\hline & Estopas \\
\hline & Lanas del canal de desagüe \\
\hline \multirow{3}{*}{ Fijado } & Agua Residual \\
\hline & Bolsas contaminadas con sustancias químicas \\
\hline & Lanas del canal de desagüe \\
\hline \multirow{3}{*}{ Neutralizado } & Agua Residual \\
\hline & Bolsas contaminadas con sustancias químicas \\
\hline & Lanas del canal de desagüe \\
\hline \multirow{3}{*}{ Suavizado } & Agua Residual \\
\hline & Bolsas contaminadas con sustancias químicas \\
\hline & Lanas del canal de desagüe \\
\hline $\begin{array}{c}\text { Esponja } \\
\text { (Desgaste } \\
\text { textil) }\end{array}$ & $\begin{array}{l}\text { Esponja contaminadas con sustancias } \\
\text { químicas }\end{array}$ \\
\hline $\begin{array}{l}\text { Sand blasting } \\
\text { (Aerografía) }\end{array}$ & Bolsas contaminadas con sustancias químicas \\
\hline Centrifugado & Agua residual \\
\hline Secado & - \\
\hline
\end{tabular}

Posterior a la identificación de los residuos producidos, se realizó la clasificación según las características de los insumos y la normatividad ambiental, de la misma forma como se procedió con los residuos de las litografías. Como resultado, todos los residuos aquí producidos son de características peligrosas, siendo los RESPEL más comunes: Y12 (Desechos resultantes de la producción, preparación y utilización de 
tintas, colorantes, pigmentos, pinturas, lacas o barnices), Y34 (Soluciones ácidas o ácidos en forma sólida) y Y35 (Soluciones básicas o bases en forma sólida).

Para el residuo originado en los procesos de lavado de las prendas, correspondiente a las lanas acumuladas en los canales de desagüe de las tintorerías, no fue posible identificarlo como residuo peligroso por entrar en contacto con los productos químicos utilizados a lo largo de la manipulación y el procesamiento de los textiles; por tal razón se recomienda adelantar pruebas de toxicidad según el Decreto 4741 de 2005 en laboratorios acreditados por IDEAM para identificar las necesidades de gestión como residuo ordinario o peligroso.

\section{Manejo interno de RESPEL en el subsector textil}

En las industrias textiles no es usual que se lleve a cabo la separación en la fuente de los residuos generados (solo el $25 \%$ de las empresas lo hace) y por lo tanto, la gran mayoría entrega estos desechos peligrosos mezclados con los residuos comunes o domiciliarios a la empresa de aseo urbano.

En el proceso de tratamiento del textil de las empresas visitadas, se emplean diversos compuestos químicos, que se aplican garantizando la dosificación precisa de cada componente, para lograr la calidad exacta del lote en elaboración.

Se encontró que el $63 \%$ de las tintorerías, usan recipientes plásticos reutilizables para la distribución de materias primas, mientras que el $34 \%$, generan residuos de bolsas plásticas contaminadas (no reutilizadas), que posteriormente se depositan junto con los residuos ordinarios.

En la etapa de desgaste de algunas prendas, las esponjas empleadas pasan a ser un residuo peligroso al tener contacto con sustancias químicas como permanganato de potasio u otras. Igualmente, son dispuestas en su totalidad como residuos sólidos ordinarios, sin ningún proceso previo.

Actualmente, sólo el 22\% de las tintorerías utilizan las piedras pómez para el desgaste de la prenda, debido a que el proceso se ha mejorado con el uso de enzimas biodegradables que no generan residuos.

El 75\% de las tintorerías en general, no poseen un área específica que cumpla con los requerimientos normativos para el acopio temporal de los residuos peligrosos que generan y por esta razón en algunos casos, se almacenan a la intemperie, cerca de fuentes de calor como las máquinas lavadoras, al igual que los recipientes plásticos vacíos donde se distribuyen los insumos; situación que puede generar riesgo porque estos envases y residuos se encuentran impregnados de sustancias con características reactivas e inflamables.

La mayoría (63\% de las tintorerías) no tiene formulado un Plan de Gestión Integral de Residuos o Desechos Peligrosos conforme se estipula el Artículo 10 del Decreto 4741 de 2005 en las obligaciones del generador. Se estima que las empresas que tienen formulado este plan de gestión, producen más de
$30 \mathrm{~kg} / \mathrm{mes}$ de desechos peligrosos, sin embargo dicho valor no se encuentra registrado, pues no se dispone de mediciones históricas que den cuenta de la realidad de generación. Aún cuando se cuenta con Plan de Gestión, se identifican también deficiencias en su implementación relacionadas con prácticas inadecuadas de separación en la fuente, almacenamiento y disposición final.

Por otro lado, sólo dos tintorerías (25\% de las entrevistadas) diligencian la información del Registro de Generadores de Residuos Peligrosos a través del Registro Único Ambiental (RUA); al no llevarse cuantificación de generación de RESPEL, no es posible establecer si las demás empresas requieren estar registradas ante la autoridad ambiental. Se requiere implementar un sistema de cuantificación.

A pesar de las debilidades encontradas, el subsector ha tratado de realizar cambios tecnológicos en su proceso productivo (63\% de las empresas), a través de la compra de máquinas lavadoras, máquinas secadoras y centrífugas de mayor capacidad y de menores tiempos de los periodos de lavado o secado, con el consecuente ahorro en el consumo de materias primas como el agua.

Igualmente han adquirido tecnología de punta como máquinas de ozono y máquinas láser, para remplazar y optimizar los procesos realizados con químicos y con trabajo manual, a fin de conseguir estandarizar y mejorar los acabados en las prendas. Otras empresas han automatizado las calderas y han instalado reguladores en las máquinas lavadoras para disminuir el consumo de energía.

A diferencia de las litografías, las tintorerías de mayor tamaño (25\% de las empresas), han capacitado al personal de procesos en gestión de residuos peligrosos, en promedio tres veces al año; dichas capacitaciones se realizan a través de un asesor ambiental con el fin de mantener al equipo de trabajo informado sobre las prácticas de manejo seguro de RESPEL en las etapas de recolección, almacenamiento y entrega al gestor externo.

Algunas tintorerías (37\%) consideran que en el mercado puede haber productos más eficientes para su proceso, como son los suavizantes -basados en siliconas y las enzimas para desgastar las prendas, sin embargo no se utilizan debido a los elevados costos.

2. Estrategias de producción más limpia en los subsectores litografías y tintorerías

De a cuerdo a los resultados hallados en la caracterización de los procesos de las litografías y tintorerías y los residuos peligrosos -RESPEL que se generan, se presenta a continuación algunos planteamientos de buenas prácticas y cambios tecnológicos que se pueden desarrollar para prevenir y minimizar la generación de tales desechos, además de contribuir a la adecuada gestión de los resultantes. 


\section{Buenas Prácticas}

- Implementar la separación de residuos en la fuente o sitio de generación para evitar la mezcla de residuos peligrosos con residuos ordinarios (orgánicos reciclables, no reciclables y biodegradables; residuos aprovechables como chatarra). La mezcla de los desechos incrementa el volumen y el peso de los residuos a disponer como residuo peligroso, ya que solamente por haber estado en contacto con el RESPEL, cualquier otro desecho o elemento se contamina y adquiere las características de peligrosidad de aquel con el cual estuvo en contacto.

Para implementar la separación es importante propiciar programas de capacitación que incentive la participación de todo el personal en definir alternativas para minimizar los residuos peligrosos en su entorno laboral, reconociendo la necesidad del manejo especial de dichos residuos resultantes del proceso productivo; de igual modo se deberán identificar y adecuar áreas específicas para la separación en la fuente y para su almacenamiento temporal.

- Seleccionar proveedores que ofrezcan planes de devolución posconsumo de recipientes o contenedores (plásticos o metálicos), para que se de manejo adecuado a los que contuvieron sustancias de características peligrosas y/o se promueva la reutilización en el almacenamiento de la misma sustancia. La devolución posconsumo de recipientes, evita que sean empleados para almacenar agua, alimentos $u$ otras sustancias que puedan contaminarse.

Mantener los contenedores y envases herméticamente cerrados, cuando se trate de productos químicos o colorantes, para evitar pérdidas innecesarias de materias primas por evaporación, derrames y por ello generación de residuos peligrosos por deterioro, vertimientos o escape de sustancias.

- Elaborar y aplicar formatos de control de los consumos de materias primas y la generación de residuos en todas las líneas del proceso de producción. Esto permite tomar decisiones sobre aquellas actividades que generan mayor consumo y producción de RESPEL y con ello la posibilidad de formular estrategias de reducción en la fuente, con el consiguiente beneficio de disminución de los impactos sobre el medio ambiente.

- Realizar un inventario periódico de las materias primas o insumos usados en cada etapa de los procesos productivos, que involucre inspección de los envases y las fechas de vencimiento, con la finalidad de consumir a tiempo los productos con fechas próximas de vencimiento, evitando la pérdida de materiales por alteración en su composición y por ello se conviertan en residuos que requieren de manejo especial. Esta acción evita desperdicio de insumos y gastos adicionales por el pago a un gestor externo autorizado para la recolección y disposición segura de dichos elementos.

En el almacenamiento de materias primas, se deben separar a una distancia prudente, los insumos químicos que puedan reaccionar al entrar en contacto, con objeto de evitar accidentes y de ello, generar más residuos

- Definir un espacio dentro de las empresas para el almacenamiento temporal de los residuos peligrosos, en un área específica que cumpla con aspectos básicos de contención, señalización y etiquetado. Debe acompañarse de formatos de ingreso y salida de residuos para llevar control sobre la generación (procedencia, tipo y cantidad) y sobre la gestión de los mismos (tipo, cantidad entregada y gestor).

Los residuos deben estar almacenados guardando las distancias apropiadas para evitar reacciones entre desechos químicos que puedan causar accidentes o emergencias. La ubicación debe hacerse según su clasificación por tipo de residuo, utilizando estantes que permitan la separación según la compatibilidad de ellos. Es de utilidad elaborar cuadro de compatibilidad.

- Llevar cuadro de registro o relación mensual de los residuos peligrosos originados, especificando procedencia, cantidades $(\mathrm{kg})$ y tipo de residuo, con el fin de determinar los puntos críticos de generación de RESPEL en el proceso productivo. Lo anterior permite determinar la trazabilidad de la gestión al comparar ésta información con lo reportado por el gestor de RESPEL en el momento de recoger estos desechos.

Así mismo, facilita la entrega de datos fiables de gestión de residuos a la Autoridad Ambiental, si lo solicita y en caso que le aplique (por cantidad generada), le permite diligenciar de manera correcta el registro de generadores: RESPEL o RÚA, según código CIIU de la empresa.

- Evaluar los programas de minimización de residuos implementados en las empresas, identificando si han logrado una disminución en la generación de desechos; realizando el seguimiento continuo de los aspectos positivos y negativos por adoptar cambios en el proceso y prácticas de manejo y/o tecnologías, ya que esto permite reconocer falencias de tales acciones y tomar medidas correctivas. En el orden, las correcciones deben documentarse, socializarse con el personal y adoptarse.

- Establecer un programa de mantenimiento preventivo a equipos, que incluya la inspección y limpieza periódica, teniendo en cuenta las recomendaciones del fabricante. Se evitará así, la limpieza innecesaria de maquinaria, que contribuye a la generación de otros residuos.

Sector Litográfico.

- El sector litográfico, debe apoyarse en una buena planificación al momento de llevar a cabo el desarrollo de 
las impresiones, realizando la mayor cantidad posible de impresiones del mismo color, con objeto de reducir o evitar la limpieza intermedia de las máquinas litográficas, lo que reduce el consumo de insumos en la limpieza de maquinaria, el tiempo de impresión y la generación de residuos.

- Identificar paños y telas de mayor capacidad de absorción para que sean reutilizados cuantas veces sea posible en las labores de limpieza de máquinas litográficas.

- Estudiar insumos que sustituyan los solventes tóxicos utilizados en la limpieza de la maquinaria, no solamente por evitar la generación de RESPEL, sino también por el riesgo que representan para la salud de los operarios que están en contacto permanente con estos.

- En tiempos de receso de producción litográfica, se deben asumir prácticas de manejo de los insumos, como emplear aerosoles para que las tintas no se sequen en los depósitos y tinteros e inhabilite su uso posterior.

\section{$\underline{\text { Sector Tintorerías }}$}

- En las tintorerías, para disminuir la generación de residuos peligrosos se recomienda que en vez de utilizar bolsas plásticas para envasar las dosificaciones de los insumos químicos del proceso, se usen recipientes plásticos para su reutilización en varias dosificaciones del mismo insumo; de esta forma se reduce la cantidad de residuos plásticos por proceso, que demandan manejo especial. Del mismo modo se controla el desperdicio de materias primas.

- Debido a la poca gestión ambiental realizada en el subsector de tintorerías, se recomienda la asignación de un responsable dentro de la empresa para ejercer funciones de supervisión de las actividades de manejo de los residuos peligrosos, apoyar actividades orientadas a la protección ambiental que incluya la caracterización y cuantificación de los RESPEL generados; realizar la gestión adecuada de los mismos, preparar la información, conservar soportes de los gestores y elaboración de indicadores ambientales, lo cual le permitirá formular y posteriormente implementar un plan de gestión de RESPEL.

\section{$\underline{\text { Cambios o mejoras tecnológicas }}$}

- Se resalta la impresión OFFSET digital que a diferencia de los procesos de impresión convencionales no necesita de procesos intermedios de pre-prensa, debido a que no requiere de la utilización de películas ni planchas para el proceso de impresión porque la imagen se crea directamente a partir de los datos digitales [14].

- $\quad$ La CTP (Computer to Plate) es una tecnología utilizada en la etapa de pre-prensa, que sirve para imprimir planchas litográficas desde el ordenador sin necesidad de utilizar películas y líquido fijador. Esta tecnología es ideal para la impresión de periódicos y elimina los costos asociados al tratamiento de residuos [15].

- En las litografías se utilizan máquinas impresoras de cuatro colores para optimizar el proceso de impresión. Esta tecnología permite disminuir el consumo de insumos, los tiempos de impresión y la generación de residuos peligrosos, debido a que se reduce significativamente el tiempo de lavado de las prensas. Lo contrario pasa con las máquinas de uno y dos colores que necesitan ser lavadas frecuentemente para el cambio de tintas.

- En el subsector de tintorerías se reconoce la tecnología de ozono, diseñada para lavado y desgaste del color de las prendas, minimizando considerablemente el uso de químicos hasta de un $85 \%$ y reducción de consumo de agua y energía hasta un $65 \%$. El sistema es autosuficiente porque toma el aire de la atmósfera y genera su propio oxígeno para la producción de ozono [16].

- La aplicación de la tecnología láser sirve para el acabado de productos textiles, debido a que reproduce fácilmente imágenes o efectos sobre el tejido, variando su color, textura y resistencia dependiendo de la cantidad de energía aplicada [16]. Esta tecnología se utiliza para grandes producciones; requiere de un trabajador por turno para operarla [17].

- La tecnología de las nanoburbujas revolucionan el proceso de suavizado y el control de encogimiento de las prendas, sin generar vertidos al medio ambiente debido a que ahorra hasta un $98 \%$ de agua [17]. En esta tecnología, el aire de la atmósfera se introduce en un reactor, que crea las nanoburbujas en una corriente de aire húmedo; ellas son responsables de transferir las propiedades de los químicos a las prendas, mientras que el aire húmedo permite controlar el encogimiento [16].

\section{CONCLUSIONES}

- El subsector de litografías se ha acercado más a la Gestión Integral de los Residuos Peligrosos, debido a que el $82 \%$ de estas tiene formulado el Plan de Gestión Integral de RESPEL, en comparación con el subsector de tintorerías, donde sólo el $25 \%$, tiene formulado e implementado el PGIR-RESPEL.

- Aunque algunas empresas de ambos subsectores tienen formulado el plan de gestión de residuos peligrosos, varias no lo han implementado de forma correcta, pues se evidencia disposición inadecuada de los desechos, sin separación en la fuente y manejo como residuo ordinario. Es claro que los planes pueden presentar deficiencias en su formulación, no han sido socializados a los empleados para su apropiación o no han sido implementados. 
- La generación de residuos peligrosos es el subsector de tintorerías es menos significativa en comparación con el subsector de litografías, debido a que los residuos más representativos son las bolsas plásticas impregnadas de sustancias químicas; algunos recipientes en los que llega el insumo, son devueltos al proveedor.

- Para adoptar las estrategias de producción más limpia referidas en éste documento, se requiere del compromiso de los propietarios y de la alta gerencia, ya que no solo se trata de la modificación de algunas prácticas, sino de cambios en los procesos productivos. Igualmente se requiere de monitoreo y control de los beneficios encontrados por la aplicación de prácticas de producción más limpia, pues en los pocos casos que se han llevado a cabo, se carece del seguimiento a los beneficios y, no es posible identificar si realmente representaron mejoras económicas, de eficiencia, de calidad e imagen para la empresa.

- La aplicación de cambios y mejoras tecnológicas para los subsectores de litografías y tintorerías implica una inversión económica por parte de las empresas, que algunas no están en condiciones de asumir, por los altos costos de las mejoras y por la variación de la actividad productiva, pues se labora a pedido y con periodos en el año de poca producción, que les representa bajos ingresos.

\section{RECONOCIMIENTOS}

Los autores del artículo quieren expresar su agradecimiento a la Corporación Autónoma Regional de Risaralda -CARDER por el apoyo financiero y técnico brindado para llevar a cabo la investigación; así mismo, a las empresas participantes por su interés y disponibilidad en suministrar la información.

Particularmente, se agradece a las administradoras ambientales Yeniré Alejandra González y Adriana Marcela Ruiz, por su apoyo en el desarrollo del proyecto como integrantes del semillero de investigación en Gestión Integral de Residuos Sólidos adjunto al Grupo de Investigación en Agua y Saneamiento de la Facultad de Ciencias Ambientales de la Universidad Tecnológica de Pereira.

\section{REFERENCIAS}

[1] Ministerio de Ambiente, Vivienda y Desarrollo TerritorialMAVDT. Decreto 4741 de 2005. "Por el cual se reglamenta parcialmente la prevención y manejó de los residuos o desechos peligrosos generados en el marco de la gestión integral". 2005.

[2] Ministerio de Ambiente, Vivienda y Desarrollo TerritorialMAVDT. Gestión integral de residuos o desechos peligrosos. Bases conceptuales. 2007.
[3] D. Escobar. Situación actual de los residuos peligrosos y RAEE en Colombia. En: XII Congreso de residuos sólidos, Basura cero. Armenia, Colombia. 2012.

[4] J. J. Martínez \& J. Rozo. Desafíos y amenazas. Consideraciones en torno a las políticas públicas de agua potable y saneamiento básico y a la gestión de residuos peligrosos. Grupo Sala, Soluciones ambientales para Latinoamérica. Editora Géminis LTDA. 2012.

[5] Corporación Autonóma Regional de Rirasalda CARDER. Plan Departamental de Residuos Peligrosos (RESPEL). 2009.

[6] Ministerio de Ambiente, Vivienda y Desarrollo TerritorialMAVDT. Política Ambiental para la Gestión Integral de Residuos o Desechos Peligrosos. Bogotá. 2005.

[7] A. A. Soler Riaño. Alternativas de producción más limpia en la gestión integral de residuos peligrosos generados en la base aérea caman. Bogota D.C. 2006.

[8] Programa de Naciones Unidas para el Medio Ambiente (PNUMA). Usando producción más limpia para facilitar la implementación de los acuerdos ambientales multilaterales. Organización de las Naciones Unidas (ONU). 2003.

[9] B. Alba Rodriguez \& O. Vargas Padilla. Formulación de estrategias de producción más limpia para el sector de fundición de metales no ferrosos en el distrito capital. Bogotá D.C., Colombia. 2009.

[10] N. G. Chaparro Sepúlveda. Caracterización de residuos sólidos peligrosos de empresas del sector de artes gráficas para la identificación de opciones de prevención y reducción. Bogotá. 2010.

[11] Comisión Nacional Del Medio Ambiente-Región Metropolitana-CNMA. Guía y control de la prevención de la contaminación Industrial. . Santiago. 1999.

[12] Centro Nacional de Producción más Limpia de Honduras-CNP+LH, C. N. Guía de Producción Más Limpia para la Industria Textil. Tegucigalpa. 2009. Recuperado el 23 de Abril de 2013, de http://www.mirahonduras.org/pml/docs/GUIA\%20DE\%20P+ L\%20TEXTIL.pdf.

[13] Ministerio de Medio Ambiente, Vivienda y Desarrollo Territorial y FUNDES Colombia. Guía de Buenas Prácticas para el Sector Textil. 2009. Recuperado el 20 de Junio de 2013, de http://www.minambiente.gov.co/documentos/textiles.pdf.

[14] Hewlett-Packard Company. Notas técnicas - Impresión offset digital en color. 2002. Obtenido en: http://www.grafikar.com/Preimpresion/Archivos\%20para\%20 Indigo/HP\%20Offset\%20digital.pdf 
[15] Agfa Corporation. (S/A). Introducción a impresión del ordenador a la plancha. Recuperado el 20 de Octubre de 2013, en:

http://www.agfagrafico.es/uploads/20101015/AGFA_Guia_de introduccion_a_CTP.pdf.

[16] Recolquim. Sostenibilidad: La clave para los procesos en prendas . XXI Congreso Latinoamericano de Química Textil. Medellin. 2013.

[17] Jeonologia. Jeanologia Science of finishing. 2013. Recuperado el 27 de Octubre de 2013, en: http://www.jeanologia.com. 